\title{
APPLICATION OF ROBOTIC AND MECHANIZED WELDING UNDER DISTURBING FACTOR CONDITIONS
}

\author{
E.V. SHAPOVALOV, V.V. DOLINENKO, V.A. KOLYADA, T.G. SKUBA and F.S. KLISHCHAR \\ E.O. Paton Electric Welding Institute, NASU \\ 11 Kazimir Malevich Str., 03680, Kiev, Ukraine. E-mail: office@paton.kiev.ua
}

\begin{abstract}
The work studies the main problems of automation of processes of multi-pass MIG/MAG welding of large-dimension parts in all spatial positions under conditions of low repeatability of assembly operations. Necessity is shown and, at the same time, insufficiency of application of only laser-television sensor for adapting purpose. A procedure was proposed for equipping a robot-technical (mechanized) welding complex with the computer vision means such as a system of laser-television and video-pyrometric sensors. The results of development of technical means, algorithmic and software support of adaptive welding complex are presented. Proposed control algorithms use the results of on-line measurement of geometry parameters of preparation of butt joint groove as well as position of molten pool. It is shown that the adaptive robotic system performes all the basic functions, typical for it, namely adjustment of electrode position and parameters of welding mode under disturbing factor conditions, and is capable to provide necessary geometry and mechanical characteristics of the weld. As an example, this work uses a robotic complex of ABB company, including welding robot ABB IRB-1600, equipped with laser-television and video-pyrometric sensors, and arc power source ESAB Aristo MIG 5000I. The welding experiments showed that the developed software and hardware allow adapting robotic technical complex for application in welding of butt joints under effect of disturbing factors such as gap size change, distortion of electrode wire, ambient temperature change etc. 8 Ref., 6 Figures.
\end{abstract}

Ke ywords : welding robot, manipulator, geometry and technical adapting, automatic control system, operator interface

Application of the robotic technical complexes (RTC) in the automatic control systems of arc welding of large-dimension thick-wall parts allows increasing efficiency and ensuring necessary level of quality of welded joint formation. Therefore, the developments aimed at determination of the possibilities of application of series mechanical multilink manipulators of welding torch and part are relevant for realizing a concept of unmanned welding technologies. A constricting factor of RTC application is insufficient accuracy of repeatability of assembly operation in butt joint welding. It results in alternation of value of butt edges gap and varying geometry of the whole butt joint. Thus, welding process promotes additional displacements in the part caused by thermal deformations and structural transformations in the weld, that as a result complicates production of the quality welded joint.

One of the methods to remove the difficulties in the process of wide application of series RTC is their equipping with computer vision systems, i.e. laser-television and video-pyrometric sensors.

The main task of the laser-television sensors is a contact-free measurement of the geometry parameters of butt joint and transmission of their values to RTC control system. The received results are used not only for adjustment of movement trajectory of the welding torch relatively to the butt axis, but also for adaptive control of welding parameters such as arc voltage, wire feed rate and amplitude of welding torch oscillations. RTC is equipped with a laser-television sensor, capable to provide necessary geometry parameters of the weld and its mechanical properties. The main advantage of the optical sensors in comparison with the touch probes is acquiring more complete information on butt geometry that allows developing more accurate algorithms of edge fill in multi-pass welding.

However, equipping the robots with only laser-television sensors is insufficient under conditions of disturbances such as change of ambient temperature, magnetization of parts, distortion of electrode wire, thermal deformation of butt etc. The indicated factors result in change of penetration depth or displacement of welding arc (weld pool) to one of the edges of butt joint which can be a reason of insufficient penetration of the other one.

In order to minimize the effect of indicated disturbances it is necessary to equip the robot with additional sensor for direct tracking a weld pool for the purpose of on-line evaluation of weld size and its spatial position relatively to butt axis without transportation lag. For this, a special video-pyrometric sensor was developed and integrated in the adaptive RTC.

At present time, a significant attention is paid abroad to a problem of development of the systems 


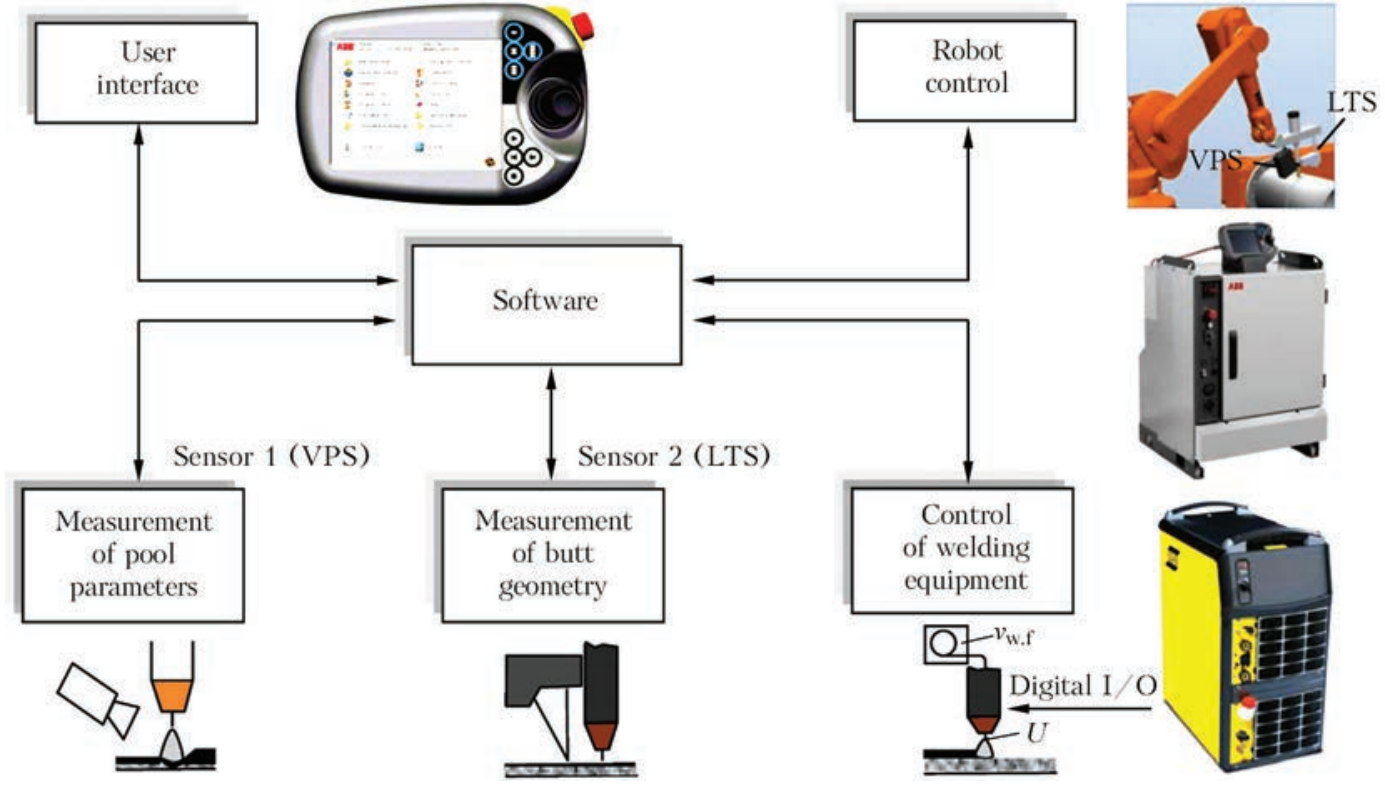

Figure 1. Flow chart of parallel double sensor system

for adaptive control of MIG/MAG welding [1-4]. However, the task has not being solved to full extent.

This work is dedicated to development of a procedure for creation of algorithmic and software support for ABB IRC5 controller of ABB IRB-1600 welding robot equipped with laser-television (LTS) and video-pyrometric sensor (VPS). LTS is used for measurement of geometry parameters of butt joints with groove preparation, and VPS is applied for evaluation of size and position of weld pool (Figure 1).

The main functions of software support of the system for automatic control of welding process parameters based on information from LTS are the following:

- regulation of welding torch movement along butt center based on actual spatial position of current butt section which is calculated on-line by LTS;

- control of shape and amplitude of torch oscillations based on current geometry parameters of the butt and, in particular, size of the gap before torch;

- control of welding mode parameters (value of electrode wire feed rate, arc voltage, welding speed), based on current geometry parameters of the butt before torch relatively to welding direction. An algorithm of calculation of values of these parameters is similar to one proposed in work [5].

Software has two levels of welding process control. Software of the upper level is realized using C\# programming language in MS Visual Studio programming environment. A program code in form of dll library files is downloaded in operator panel $\mathrm{ABB}$ FlexPendant [6] (Figure 2). Software of the lower level is developed using Rapid (ABB) language in ABB RobotStudio programming environment.

The developed software is designed for operation under control of real-time OS Windows CE.
The software is realized in form of several flows which are executed in parallel using program interrupts mechanism:

- control of robot movement (movement of sensors, mounted to last robot link) on preplanned trajectory taking into account on-line correction;

- robot controller inquiry for determination of coordinates of tool central point (TCP) current position. A value of coordinate $X$ corresponds to the position of LTS laser plane in welding direction along the butt;

- LTS on-line inquiry and receiving form it $Y$ and $Z$ coordinates of points of intercept of sectional laser plane with butt edges;

- VPS on-line inquiry for determination of coordinates of weld pool center and its geometry;

- calculation of current values of welding mode parameters and values for correction of torch position (in transverse direction and on height relatively to butt axis) and transmission of these values to robot controller.

After the dll library files, created in MS Visual Studio, are downloaded in the robot controller, the main menu of the panel in addition to the basic items starts

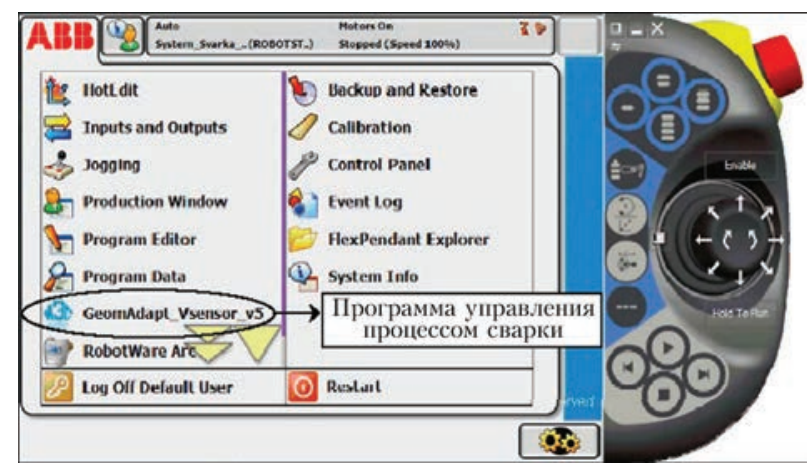

Figure 2. Main menu of operator panel ABB FlexPendant 


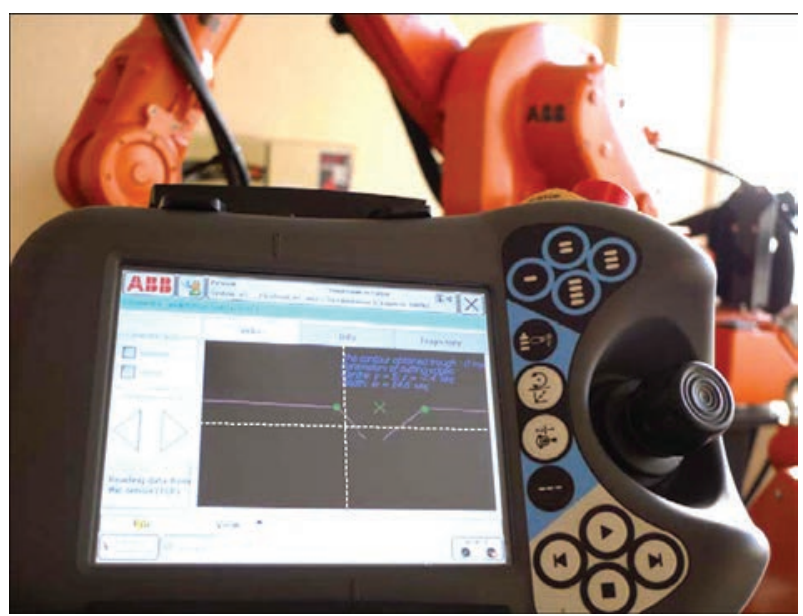

Figure 3. Main dialogue box of operator interface on screen of ABB FLexPendant panel

displaying an item, including title of a program for welding process control, for example, «GeomAdaptVsensor_v5» (see Figure 2).

This menu item launches the program of welding process control (Figure 3). A significant part of interface of dialogue box is taken by an image of laser band contour, which is dynamically changed in a process of scanning of the butt before and during welding.

In the upper part of the screen there are «Video» and «Info» inlays which contain information on butt geometry, weld pool and calculated values of torch trajectory correction, and «Trajectory» inlay is designed for displaying the butt and trajectory of torch movement. In the left part of the window there are control elements (buttons) for selection of manual («Correction manual») or automatic («Correction auto») correction mode of welding torch. Menu items «File» and «View» are located in the lower part.

Menu item «File» allows saving a real (with correction) trajectory of torch movement in a file after welding of each weld. As a result, part passport can be received, which shows all necessary information on trajectory and welding modes in all sections of root and fill welds of part being welded.

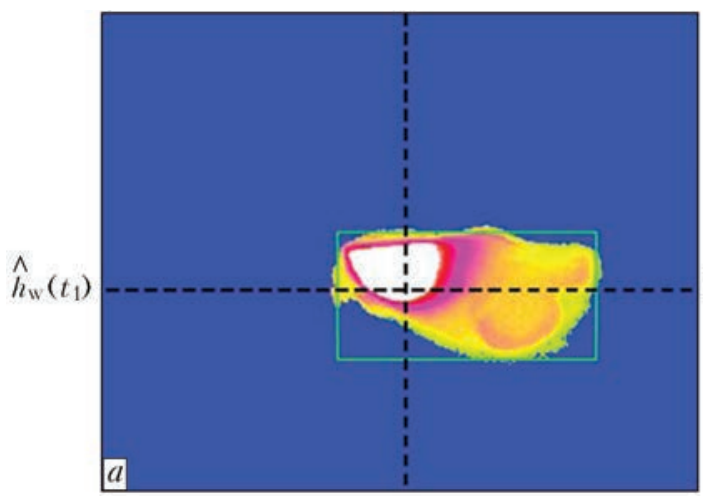

Menu item «View» provides for a possibility to display on the operator panel screen a mesh with selected step and central axes which intersect in a point with $y=0, z=0$ coordinates. Thus, the operator has a possibility of visual evaluation of butt displacement and change of its geometry in process of welding.

In addition to measurement of the butt geometry and its spatial position, a corresponding information on weld pool is also evaluated in process of welding. The latter can be displayed on the operator panel screen. Registration of the image of weld pool thermal field and estimation of height of weld pool relatively to low surface of butt groove is carried out with the help of double channel pyrometric sensor. Based on given information the RTC control system forms a correction of electrode position in process of welding and correction of welding mode parameters. Figure 4 shows an image of a thermal field of weld pool in two extreme positions of the torch, which makes transverse oscillations.

Position of a zone of maximum heating (arc burning area) is kept in the middle part of the frame, while less heated zone of the weld pool is periodically moved, that is caused by torch transverse oscillations. Stationary position of the zone of maximum heating is explained by the fact that a pyrometer sensor is mounted directly to the torch and, therefore, the arc is stable on the image, and weld pool contours are moved.

Interaction between welding robot controller, LTS and VPS is realized with the help of Ethernet technology of batch data transmission on software upper level. Figure 5 shows a structure of software for realizing the interaction between the system components. C\# MS Visual Studio programming language was used in software development.

The main functions used in software realizing are the following:

- development of end points on socket and port addresses, which correspond to IP-addresses of sensors;

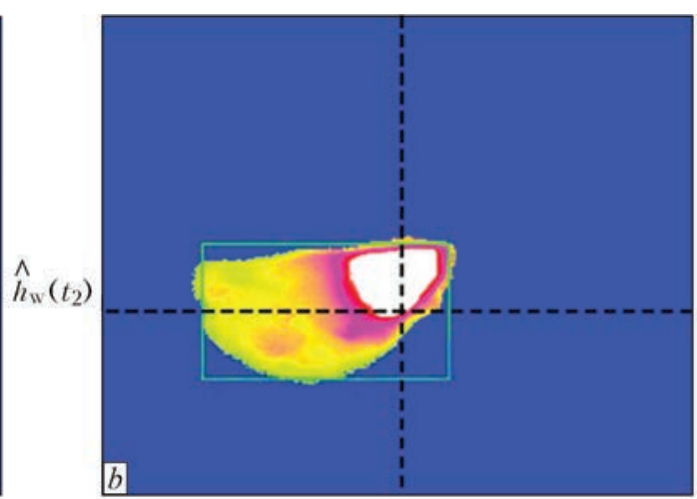

Figure 4. Image of thermal field of weld pool in welding of root weld with transverse oscillations in extreme left $(a)$ and right $(b)$ postion relatively to butt axis 


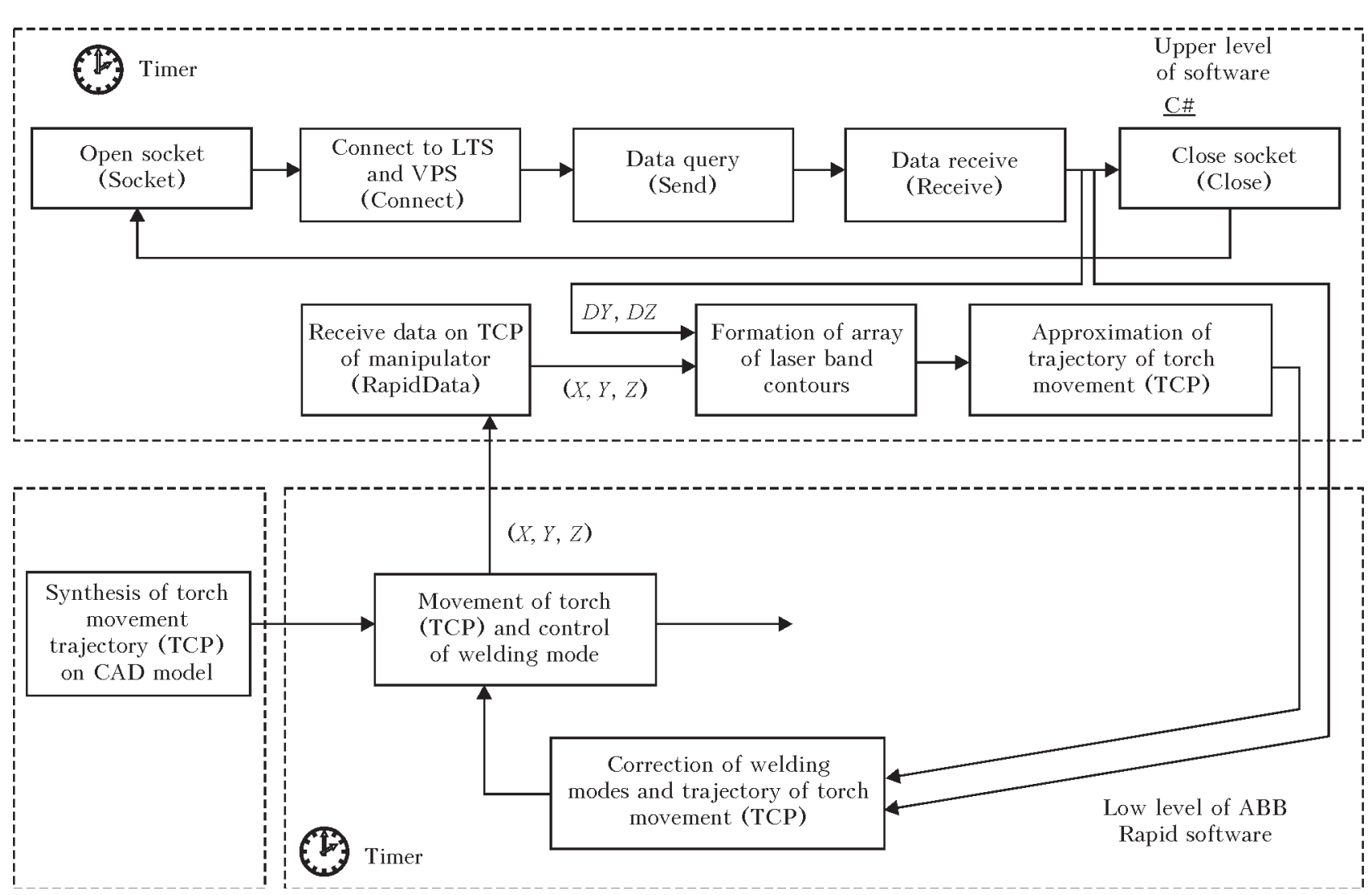

Figure 5. Flow chat of software for information transmission between system components

- development of socket TCP/IP (Transmission Control Protocol/Internet Protocol);

- tie the point to the developed socket - myscoket.Connect(ipep);

- send data via open socket - mysocket.Send$\operatorname{To}(0)$;

- wait for an answer from the sensors - Listen() procedure forms a list of coordinates of characteristic points of the laser band contour received from the sensor, which are used for determination of geometry of edge preparation and gap.

Connect working space (namespace) RapidDomain [6] in order to get access to robot data in a format of Rapid programming language from $\mathrm{C \#}$ program. This document describes PC SDK classes with different types of Rapid data. RapidData-object, which is linked to corresponding data, is created for reading and recoding of RapidData.

The following steps of algorithm are performed in order to transmit the values of the corrected coordinates of welding torch to the low level program (Rapid):

- on-line read out of current coordinates;

- correction of coordinates in accordance with LTS and VPS readings;

- transmission of corrected coordinated to the welding robot controller.

The software of low level (ABB Rapid), designed for realizing adaptive correction of torch vertically and horizontally in process of welding, has the fol- lowing main structural components, namely main procedure «main()», procedure of pre-formed trajectory «Path_10» in accordance with ideally assembled welding object, and procedure «TRAP Path_10_correct», which takes into account the errors of groove preparation and assembly operations. Before procedure fetching the variables are declared, for example «hori_id» and «veri_id», which are connected to «correction generator» for performance of horizontal and vertical correction.

Receiving the data from software of upper level was carried out with the help of timer interruption mechanism. Firstly, a «timeint» variable with «intnum» data type is declared [7]. After that, the «timeint» variable with the help of CONNECT function is connected with TRAP type procedure, which in this project is titled «Path_10_correct». Besides this procedure turn on ITimer interruption timer, parameters of which indicate value of time interval between sensor sampling (for example, $0.2 \mathrm{~s}$ that equals $5 \mathrm{~Hz}$ period). «Path_10_correct» procedure performs correction of values of coordinates of goal point, to which torch is moved in process of welding of root weld, and correction of welding mode parameters.

CorrWrite function is used for displacement record (in coordinate system it is a trajectory). It results in immediate correction of coordinates of the goal point of trajectory (TorchPos) and, respectively, torch movement trajectory to corrected point. ArcRe- 


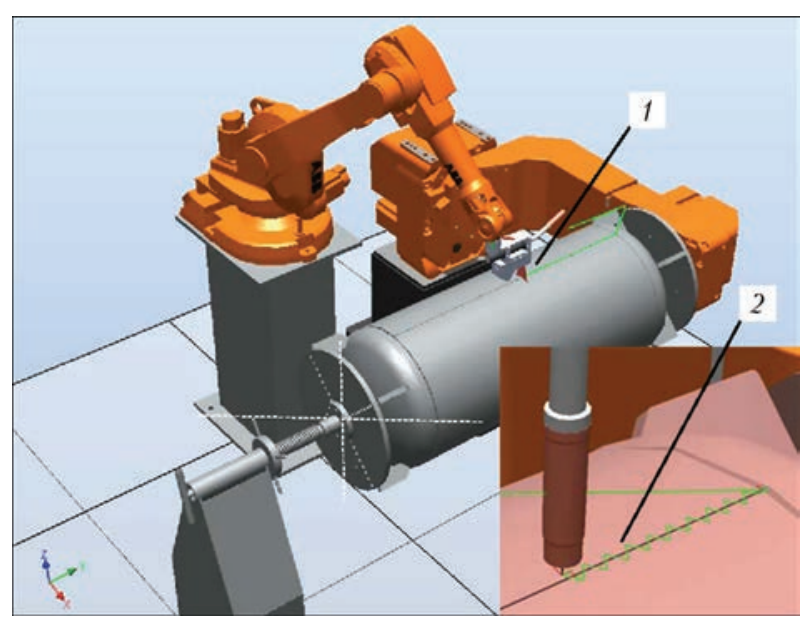

Figure 6. Simulation model of adaptive RTC: 1 - butt scanning trajectory before welding; 2 - corrected trajectory of torch movement (with oscillations)

fresh function is used for updating the welding mode parameters (adaptWd) in process of program performance [8]. Updated values of the welding parameters are transmitted to welding instructions «ArcX» that in accordance with program structure is refer to «Path_10» procedure. ArcL function [8] is used for realizing welding along linear trajectory instruction. Its first parameter is a goal point, value of coordinate of which is continuously corrected using interruption timer. Besides, rate of movement, values of welding start parameters adaptSm, values of welding mode parameters, accuracy of positioning fine, parameters of working tool MyNewToll_Torch_Esab and working objects Workobject_truba are indicated. The latter parameter indicates that the goal point is not constant.

RTC simulation model was constructed for checking the correctness of operation of algorithmic and software support (Figure 6). The model allows reconstructing the main structural peculiarities of the complex, i.e. kinematics of robot, part positioner and their mutual location, geometry of part being welded with butt groove preparation, design of welding torch, LTS and VPS. Also, the model allows recreating all the steps of technological process, starting from launch of control program performance with the help operator virtual panel and ending with a step of on-time torch movement on the corrected trajectory along the butt (see Figure 6). The next possibilities are realized for this, namely butt scanning with the help of LTS, formation of a list of typical points of laser plane projection on the butt and calculation of welding torch movement trajectory taking into account correction.

\section{Conclusions}

1. The procedure was developed for creation of algorithmic and software support of a subsystem for stabilizing geometry of weld pool based on information from laser-television and video-pyrometric sensors. This procedure can be used for development of software of adaptive mechanisms and robotic welding complexes.

2. The approach was proposed on development of simulation 3D model, that allows reconstructing all the steps of technological process, namely scanning of butt joint, planning the trajectory of welding torch movement and direct torch movement taking into account correction on data from LTS and VPS. The developed model can be used for evaluation of work of separate system components as well as operation of complex in whole.

1. Gladkov, E.A., Brodyagin, V.N., Perkovsky, R.A. (2014) Automation of welding processes. Moscow: N.E. Bauman MGTU.

2. Fernando de Aguiar Faria, Abraao Marques Tavares, Marina Spyer Las Casas (2010) Machine vision and artificial neural networks for seam tracking and weld inspection. $A B C M$ Symp. Series in Mechatronics, Vol. 4, 768-775.

3. Xiao, Z.W., Tarn, T.-J. et al. (2010) Research on a trilines laser vision sensor for seam tracking in welding: Robotic welding, intelligence and automation. LNEE, Vol. 88, 139-144.

4. Moon, H.S., Beattie, R.J. (2002) Development of adaptive fill control for multitorch multipass submerged arc welding. Int. J. Adv. Manuf. Technol., Vol. 19, 867-872.

5. Skuba, T.G., Dolinenko, V.V., Kolyada, V.A. et al. (2013) Algorithm of technological adaptation for automated multipass MIG/MAG welding of items with a variable width of edge preparation. The Paton Welding J., 1, 14-20.

6. (2013) Robotics application manual. FlexPendant SDK RobotWare: Doc. ID 3HAC036958-001, Rev. B.

7. (2013) Robotics technical reference manual. RAPID instructions, functions and data types RobotWare: Doc. ID 3HAC16581-1, Rev. P.

8. (2013) Robotics application manual. Arc and arc sensor RobotWare: Doc. ID 3HAC16591-1, Rev. Q.

Received 26.01.2016 